\title{
The Unexpected Bioenergetic Function of Neuromelanin. Implications in the Biology of Learning and Memory
}

\author{
Arturo Solis Herrera, Ruth I. Solis Arias \\ Human Photosynthesis ${ }^{\circledR}$ Research Center, Aguascalientes, Mexico \\ Correspondence to: Arturo Solis Herrera, comagua2000@yahoo.com \\ Keywords: Hydrogen, Energy, Memory, Eukaryotic Cell, Neurons, Cerebral Cortex, Substantia Nigra \\ Received: September 8, $2017 \quad$ Accepted: November 27, $2017 \quad$ Published: November 30, 2017 \\ Copyright $\odot 2017$ by authors and Scientific Research Publishing Inc. \\ This work is licensed under the Creative Commons Attribution International License (CC BY 4.0). \\ http://creativecommons.org/licenses/by/4.0/

\section{c) (1) Open Access}

\section{ABSTRACT}

Progressive Recent Memory loss is the main complaint in Alzheimer's disease patients. There is not a simple or congruous explanation yet due to highly complex mechanisms by which the Central Nervous System store and organize experiences every day, developed along 4 billion years of evolution; thereby learning and memory are poorly understood biological processes. The finding of the unsuspected intrinsic property of melanin to transforms light into chemical energy through water dissociation, as chlorophyll in plants; opens a new and wider horizon in the form in which the CNS generate and distribute chemical energy transporting it through molecular hydrogen produced by substantia nigra of mesencephalon and other pigmented structures. The mechanisms by which human modifies their behavior through learning and memory remain a mystery despite several decades of research. However, the unexpected role of melanin molecule bioenergetics of first order, very above the chlorophyll and glucose, indicates that the study of the fascinating processes involved in learning and memory several levels, needs to be started with the study of the generation and distribution of energy neuro-melanin-releasing to all the brain. In this article, we explain succinctly, the way hydrogen molecular $\left(\mathrm{H}_{2}\right)$-gas-, from the dissociation of water by the neuro melanin, moves from its source and permeates easily through the different structures of the Central Nervous System, as well as be the smaller atom, does not combine with water, moving through it in accordance with the laws of the simple diffusion. Molecular hydrogen is the main carrier of energy that nature uses in the entire universe, so that, once the neuro melanin separates it from the water, this displaces transporting its precious cargo of energy and its remarkable antioxidant capacity capable of reduce oxygen and form water.

\section{INTRODUCTION}

Following the discovery of the unsuspected property intrinsic of the melanin transforms the visible 
and invisible light into chemical energy by means of the dissociation of the molecule of the water [1], as the chlorophyll in plants, old paradigms are breaking and appear new challenges.

Our purpose is beginning to decipher the operation of the cell based on the concept of that the true sources of energy of the eukaryote cell are the molecular hydrogen $\left(\mathrm{H}_{2}\right)$-gas- and the electrons of high energy $\left(\boldsymbol{e}^{-}\right)$that continuously are formed inside the cell, thanks to the molecule of melanin, which decompose and reform the water incessantly and orderly.

It is necessary to modify our concepts about the functioning of the eukaryote cell separating glucose as a source of carbon chains with which our body synthesizes the $99 \%$ of biomolecules, and melanin as a source of energy. This is: glucose is the universal precursor of any organic matter in our body, but cannot provide the energy that its own metabolism requires. The necessary energy to impel the highly complex intracell biochemical network is taken from visible and invisible light, through melanin's water dissociation, as chlorophyll in plants.

The challenge now is to understand the functioning of the cell's metabolic pathways based on the chemical energy that emanates from the melanin. It will be a long but interesting task because it changes completely the biology. To way of example, for this article, we will refer to the molecular logic of learning and memory, starting at the substantia nigra and locus coeruleus as main sources of energy of the Central Nervous System, making analogy in the way in which the eye-lens goes forming along the pre- and post-natal life.

\section{BACKGROUND}

The present state of art in regards neuro-melanin chemical structure and functions, is the same of the past one: uncertainty.

Neuro-melanin has for long been regarded as an uninteresting waste product of catecholamine metabolism. Lower mammals as rats or mice contain no neuro-melanin, meanwhile humans contain the highest phylogenetic levels.

It is considered that melanin normally has powerful anti-oxidant properties because it reduces ferric to ferrous iron. However, when its reductive capacity is overcome by long lasting (during life) chelated iron, or zinc, also molybdenum, or selenium, cooper or even gold; melanin converts into a pro-oxidant. Recall that in presence of metals, hydrogen will work with -1 .

Substantia nigra melanin is absent or significantly scarce in two conditions of life in which the coordination of movement is either inefficient (newborn babies) or strongly compromised (Parkinson). Neuro-melanin levels are low in infancy and then increase steadily until about $60-70$ years of age when they level off.

The lack of knowledge in the structure of neuro-melanin hampered studies on its biological function and possible role in the pathogenesis of Parkinson's disease.

Neuro-melanin is described as located in the Substantia Nigra and other dopaminergic cells group in the ventral tegmental area, in the parabrachial nucleus, in the Locus Coeruleus; however, many regions of the brain can form neuro-melanin in vitro.

Brain neuro-melanin is normally localized in discrete granules inside neuronal cell bodies, mainly in per-nuclear space. Albinos show pigmented Substantia Nigra. Neuro-melanin when bleached is like lipofuscin. In Primates, this pigment is well evident and they share with man similar neuro-melanin related disorders.

The occurrence of pigmented neurons has been reported in Amphibians too, where they may have roles like those they have within man and Primates. A lower amount of pigment, with respect to normal individual, has been found in the Substantia Nigra of phenyl-pyruvic oligophrenics.

Many putative roles have been assigned to neuro-melanin, as ion metal scavenger, phonon-electron coupler, drug scavenger and releaser, free radical sinker, bio-cybernetic function, Parkinson's disease and related conditions may be the result of oxidative stress [2]; many agents such as drugs, metals, viruses, $\mathrm{CO}$ and trauma, may produce parkinsonian symptoms; some authors found that the most vulnerable cells in 
Parkinson's disease appear to be the less rich of neuro-melanin, in spite of reports that suggest a higher vulnerability of the most pigmented neurons in the Substantia Nigra.

Also, neuro-melanin may have further functions yet unrecognized. So far very few people claim that neuro-melanin should have a positive function in the pigmented neurons physiology. A major question is: why has not neuro-melanin been discarded by natural selection? Why does neuro-melanin occur within the highest phylogenetic levels?

We believe that our finding of the unsuspected intrinsic property of melanin to transform light visible and invisible into chemical energy through the dissociation of the water molecule, like chlorophyll in plants; will mark a before and after in the history of neurology of memory.

Hydrogen as main energy carrier in neuron eukaryotic cell and learning and memory levels organization.

Mechanisms and anatomical structures involved in storing and organization of experiences in Central Nervous System, are not understood. It is already described in scientific literature minor morphological changes in some structures as hippocampus, however their role in memory is far to be clear.

The biology of learning and memory, has been investigated at a variety of levels from the cellular to the neuropsychological. The phenomena observed in neuroscience study as collateral sprouting, receptor adaptations, synaptic depression and facilitation; dendritic growth in response to enriched environment, and recovery of function after brain injury; they all reflect the nervous system's capacity of plasticity.

Therefore, arising from melanin as main energy source of CNS and molecular hydrogen as fundamental energy carrier, it is possible to build up a new coherent scheme that allows the concatenation of the generation and distribution of energy and the functioning of learning and memory, making an analogy with crystalline anatomy.

The nucleus of eye-lens

The anatomy of crystalline is a live-long lasting dynamic process. During early gestation appears embryonic nucleus localized centrally (Figure 1).

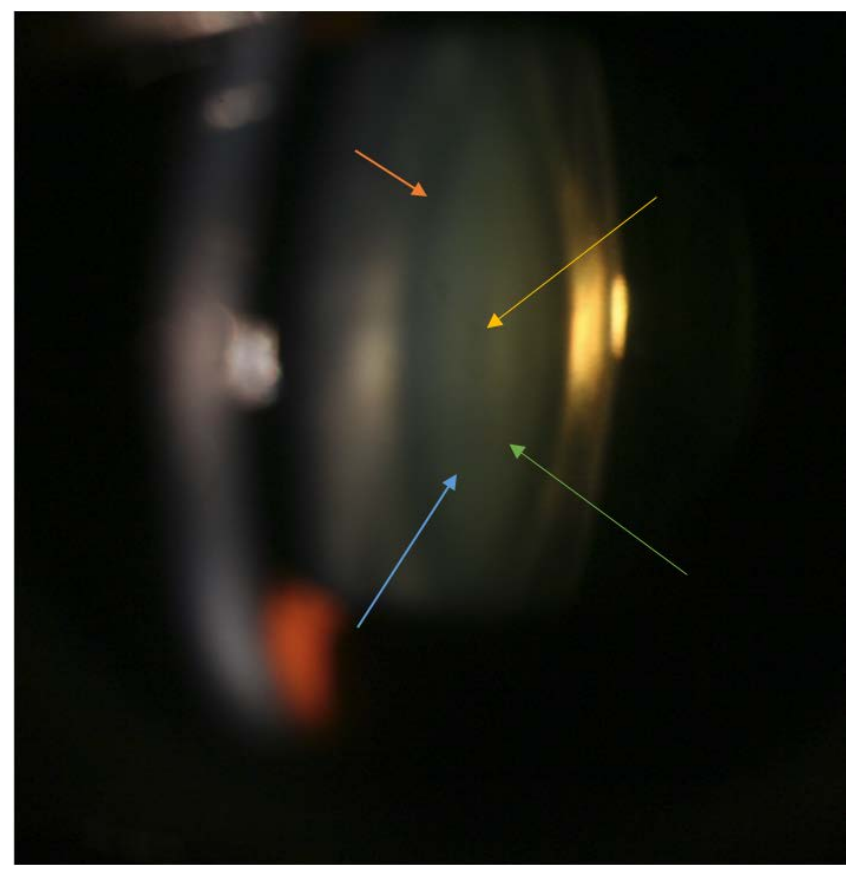

Figure 1. Macrophotography of the eye-lens in a live patient. The first nucleus that appears during gestation is embryonic nucleus (yellow arrow), followed by fetal nucleus (green arrow) forming an envelope around the previous one. The next nucleus is the childhood (blue arrow) surrounding the fetal nucleus. The last core that forms is the farthest and is called adult nucleus (orange arrow). 


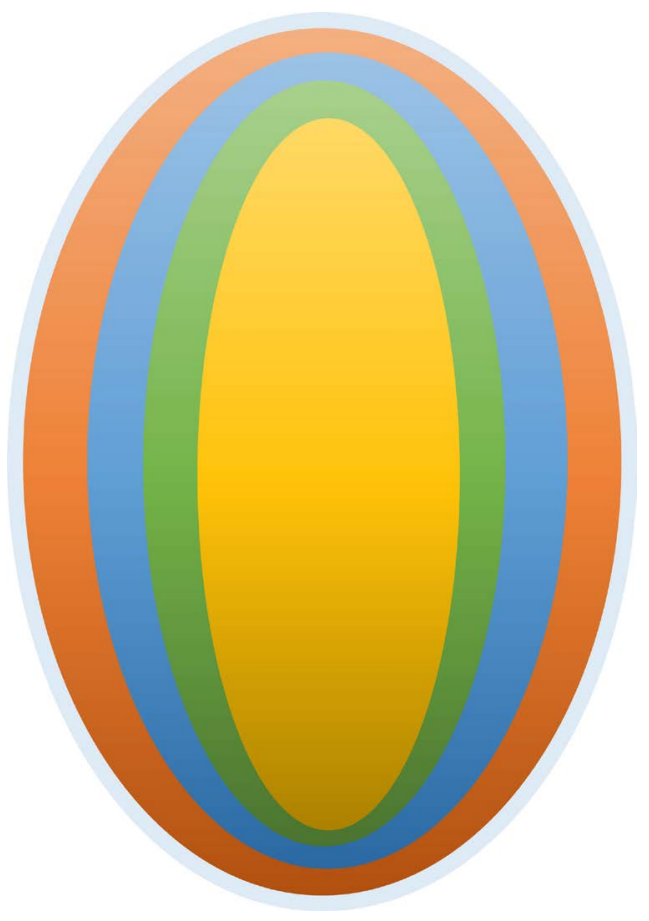

Figure 2. The scheme shows the anatomical arrangement of the different nucleus of the lens, the embryonic drawn in yellow, the fetal Green; the children and youth in blue, and the core that is form in stages adult of the life is displayed in Orange. The capsule of the crystalline, an acellular structure that surrounds to the Crystalline all her life, in clear blue.

Latter other structures or nucleus gradual and sequentially appears, as fetal, childhood, youth nucleus, and finally adulthood (Figure 2).

This anatomical arrangement invariably happens in all human beings under normal development and circumstances. The most central nucleus, is denominated embryonic by the stage in which appears, the next one is fetal nucleus; and so on. Every lens nucleus is over posed to the previous one.

This seems like the order in which memory levels appear during gestation and thereafter. The importance of the memory-related structures' location appears to reflect in its proximity to the pigmented structures. While more closely than them, most important are phylogenetically speaking. The location relatively distant formerly called limbic circuit structures, may explain why the molecular hydrogen $\left(\mathrm{H}_{2}\right)$, being the lightest element, tends to rise.

According to the time in which appear the memories, is the firmness shown in our memory. And is directly proportional, since the more appear early in life, more likely that remain during all the time of life of the subject.

If we look at the respiratory movements as a kind of memory, is clearly visible that they learn in the first few minutes of life, and last all the time. The Brainstem respiratory centers are a collection of anatomical loci within the brainstem. They also receive significant input from a variety of sensors, i.e. arterial oxygen, arterial $\mathrm{CO}_{2}$, $\mathrm{Ph}$, etc.

The respiratory centers are spread relatively diffusely throughout the medulla and pons, displaying unique functional contributions to the control of respiration. The inspiratory center is located within the dorsum of the medulla, and in absence of any afferent input, intrinsically displays a rhythmic pattern of discharge. This memory's pattern of rhythm discharge can be influenced by the input of chemoreceptors, pulmonary stretch receptors, irritant receptors, J (juxtaposed) receptors, etc.

The hippocampus, the part of the mammalian brain thought to be most directly involved in aspects of complex memory [3] and the brainstem respiratory centers have certain characteristics in common, i.e. 


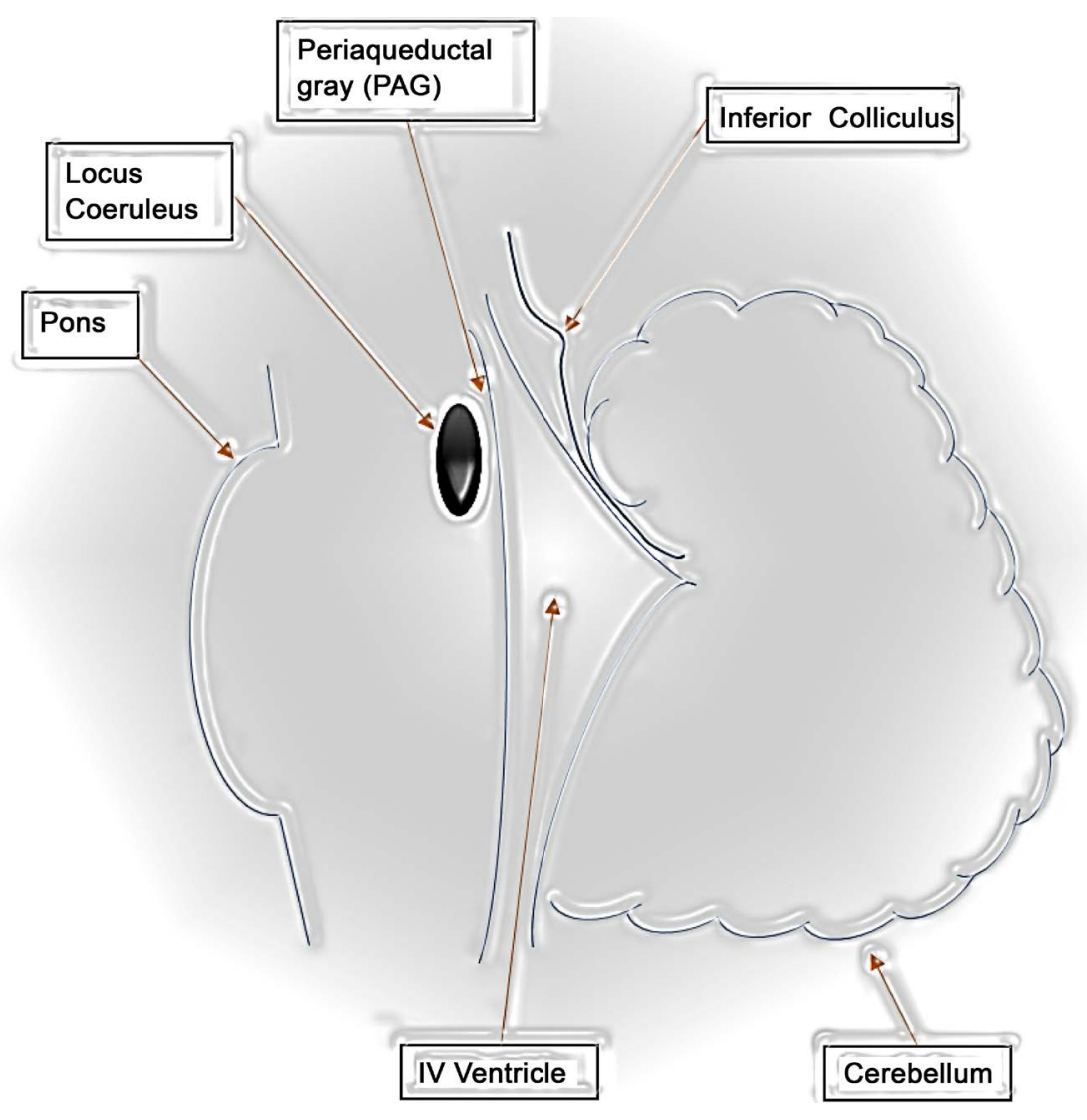

Figure 3. Diagram showing approximate location of locus coeruleus, a highly pigmented structure in brainstem.

both are located close to locus coeruleus (Figure 3) and substantia nigra.

The advancement of the study of learning's biology is remarkable, it has revealed new features of synaptic transmission, new cell-biological functions of synaptic signalization, a complex cross-talk between synapsis and cell nucleus; the intrinsic signaling properties of neurons, etc. However, the complexity of fascinating mental processes of learning and memory is still formidable.

But even the fundamental forms of learning and memory at a cellular or molecular level require energy, so far, a relegate actor in research of the molecular logic of behavior. Even the neural networks in which the hippocampus was embedded, need energy to functioning, to interact, and furthermore: to keep the shape.

The eukaryotic cell uses energy in many ways, and neuron cell is not an exception.

To bring the unsuspected intrinsic property of melanin to dissociate the water molecule, as chlorophyll in plants; to bear on study of learning, should allow us to take a very different but necessary reductionist approach. It is necessary to study the earliest instances of memory that are the generation of distribution of energy (from melanin and not from glucose).

The generation and distribution of energy from melanin is involved since simple animal models, particularly invertebrates' ones; to higher animals exhibiting neuronal organizations and neuronal mechanisms qualitatively different from those found in simple animals. However, in both cases the very first step is the same one: the dissociation of the water molecule. This is: the transformation of light into chemical energy, which is transported-after water dissociation-by molecular hydrogen $\left(\mathrm{H}_{2}\right)$, the main energy carrier in the whole universe.

The elementary forms of learning are common to all animals with an evolved nervous system, starting by the nature of the chemical energy (Hydrogen), so there must be conserved features in evolution in the 
mechanisms of learning at the cell and molecular levels.

We must keep in mind, that the chemical energy that impels the different biochemical reactions involved in learning and memory, in spite their simplicity or complexity; is in form of molecular hydrogen and high energy electrons, and happens since beginning of time in the same way.

\section{Substantia Nigra}

Greatest accumulation of melanin in primates is observed in human beings at the Substantia Nigra, that is strategically placed in both sides of mesencephalon, slightly up of pons (Figure 4).

Since melanin releases energy symmetrically, in all directions; (Figure 5), the growing spheres representing molecular hydrogen displacing follows the laws of simple diffusion, can, eventually; reach until the last corner of the neurological structures housed intra cranially.

The Substantia Nigra is a structure present on both sides of the midbrain. It is symmetrical between themselves. At birth has little pigment, and as the individual develops it will increasing pigment until it reaches a stop between 60 and 70 years, that gradually seems to vanish [4] (Figure 6).

The growing energy spheres coming from Substantia Nigra of the left side, coalesce with the growing spheres that come from the Substantia Nigra of the right side (Figure 7).

Nerve structures related to the most primitive memory, more fundamental, more vital, such as structures that manage vital aspects such as: respiratory rate, heart rate, blood pressure, etc., were developed early in evolution, and are therefore very close to the Substantia Nigra, due to pressumibly needs more energy from water dissociation.

The neural structures that is developed more belatedly, is found more remote of the Substantia Nigra and more nearby to the cerebral cortex, and are related to the recent memory. Therefore, when the Substantia Nigra decreases its efficiency in terms of transformation of visible and invisible light into chemical energy, through the dissociation of water, as chlorophyll in plants, the functions of learning and memory which are altered first are the farthest to the power source.

This overlapping creates a zone of high energy in mesencephalon and other nearby structures, where vital functions as heart beat and respiratory frequency are controlled.

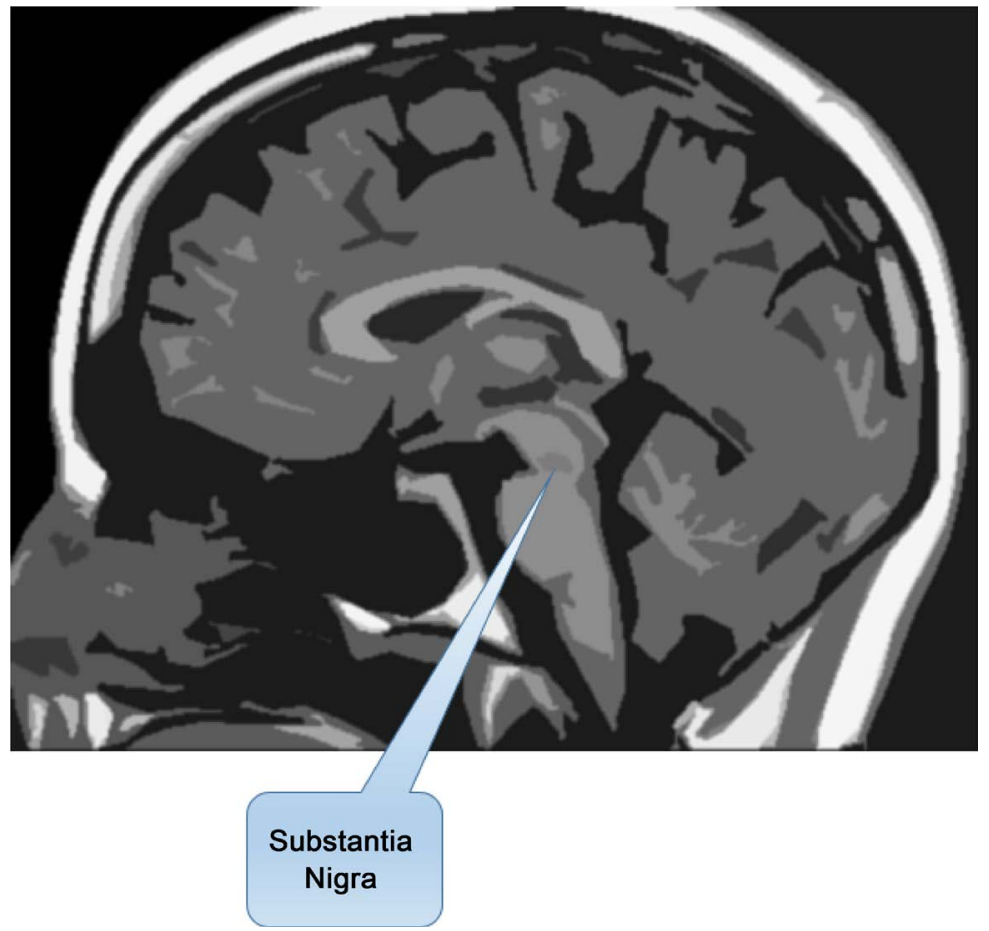

Figure 4. One of the main pigmented structures of the CNS is the Substantia Nigra, whose location is shown in the figure. 


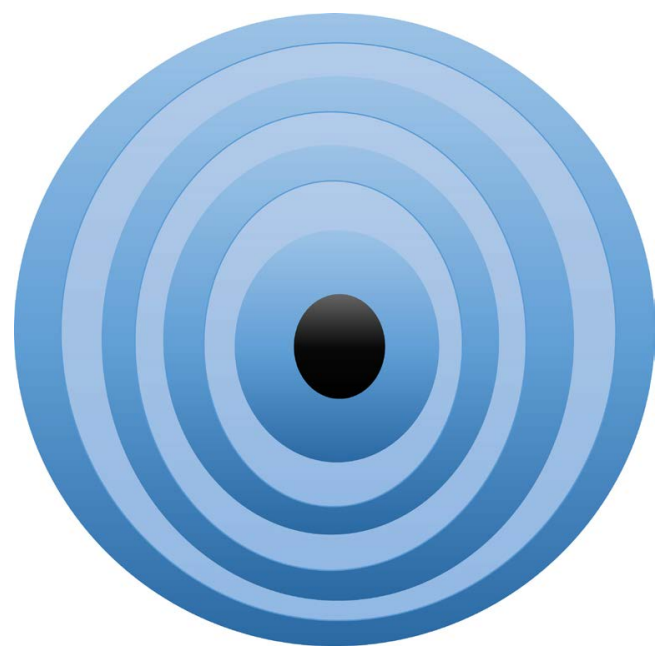

Figure 5. The energy that emanates from the melanin, representing it as growing circles of power, alternating nature; thus, each one contains higher concentration of molecular hydrogen (dark-blue), result of the dissociation of water; and alternated (pale blue) circles with the higher concentration of re-formed water and high-energy electrons. Recall that by each two molecules of re-formed water, 4 high energy electrons are generated.

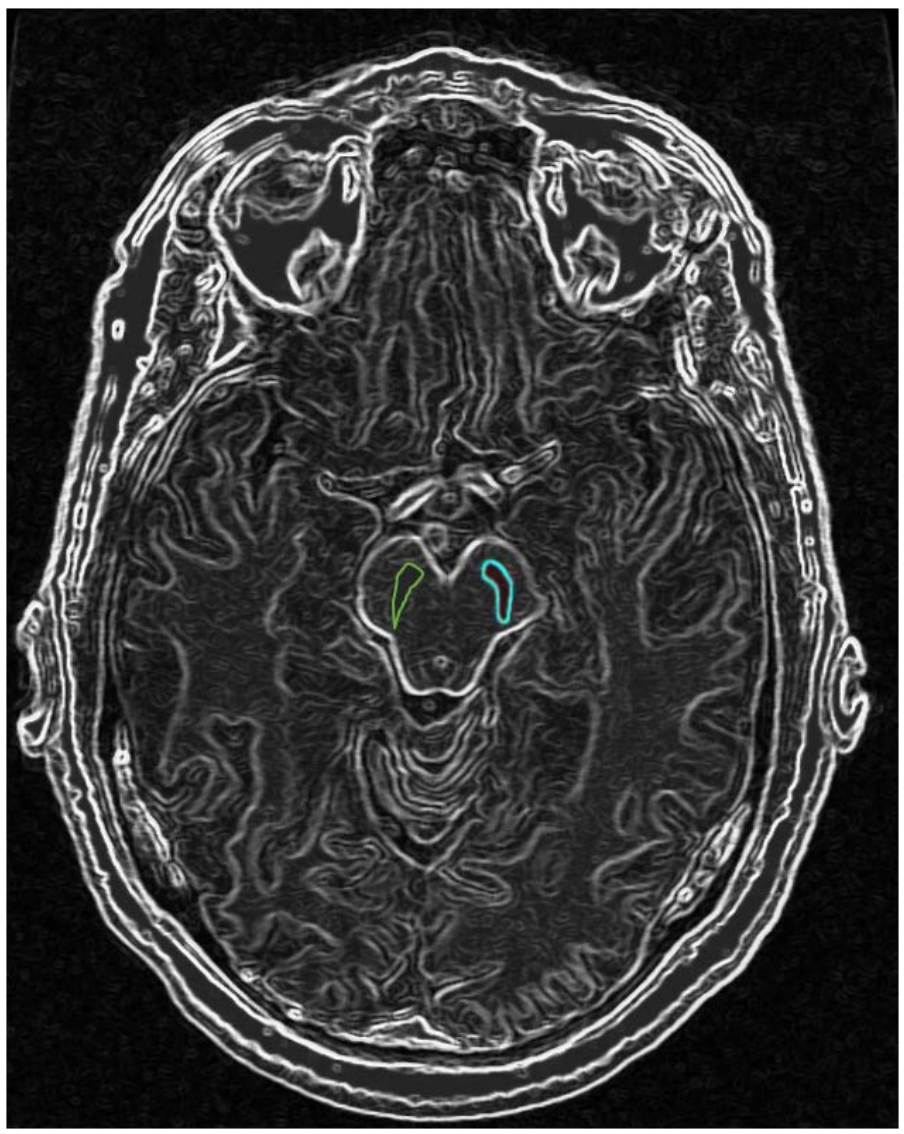

Figure 6. Scheme of the midbrain in where is draws the location of the substantia nigra on both sides of the midbrain. The basal ganglia are located above the substantia nigra, so they are not represented in the figure. 


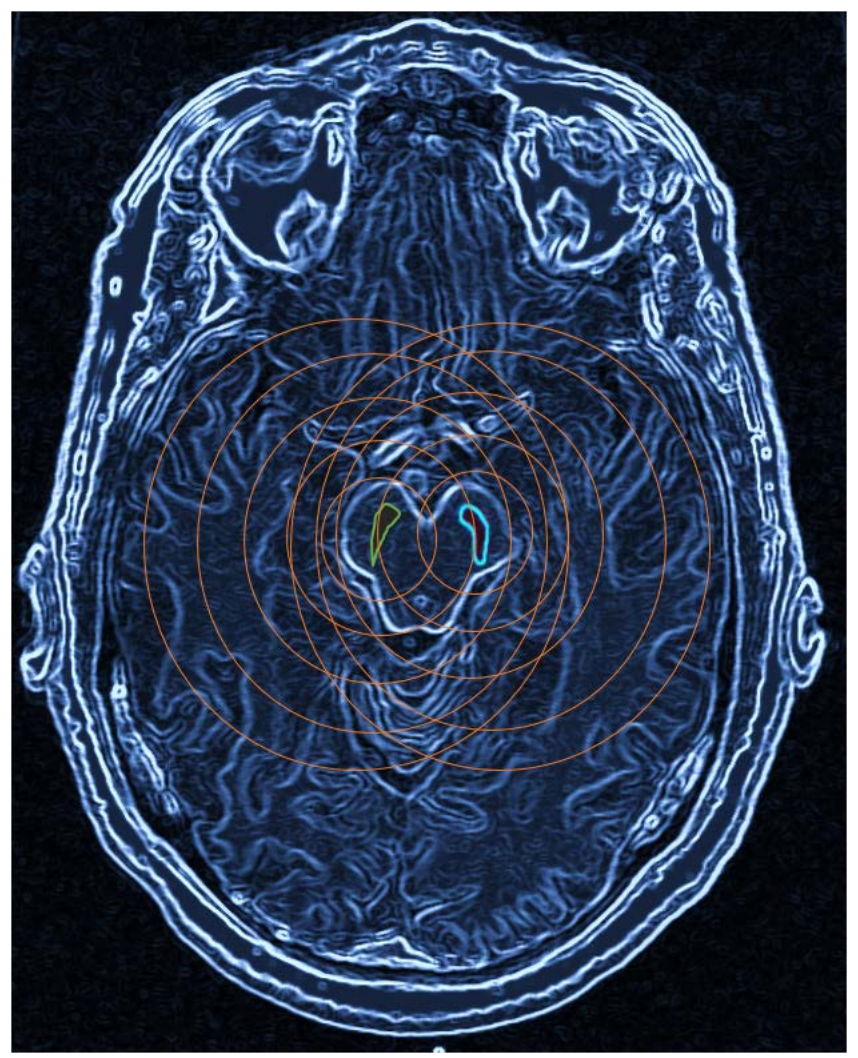

Figure 7. Growing spheres of energy coming from the substantia nigra, in the drawing are represented as circles of orange color, ranging from the Center to the periphery, following the laws of simple diffusion.

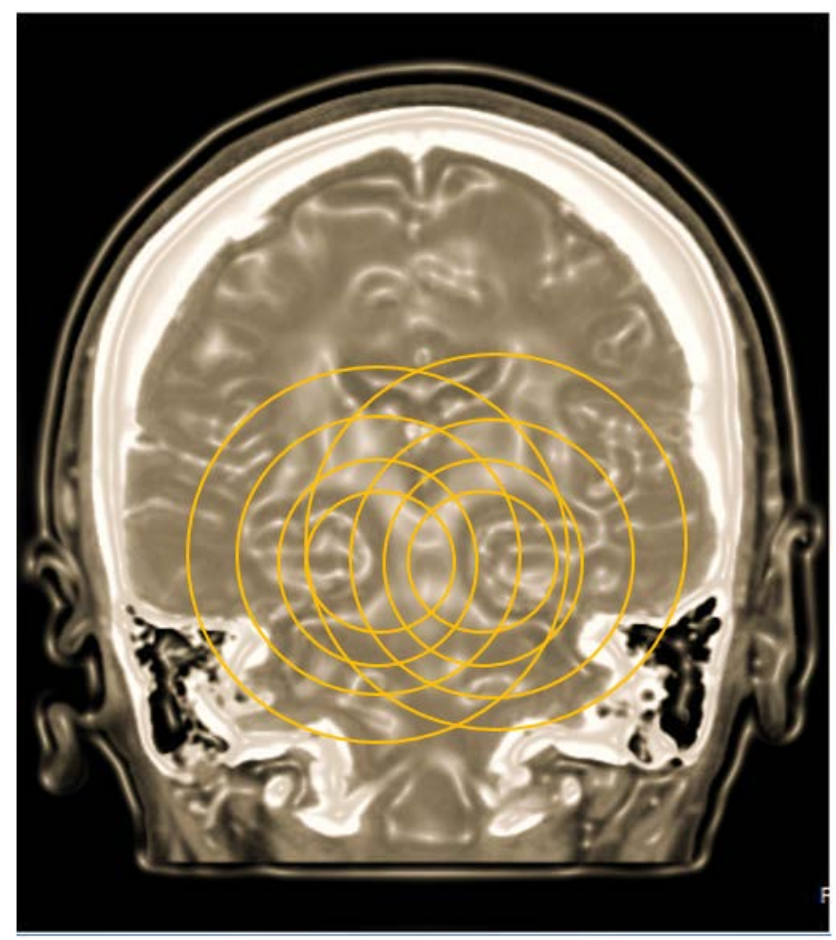

Figure 8. The growing circles of energy, are released symmetrically in all directions. 
The regulation of these vital cycles can be considered as a kind of primitive, fundamental, or vital memory, and involved structures are located closest to the source of energy, this is neuro-melanin.

The functions that sequentially appear along development of the individual, are controlled by neurological structures that surround also the primary source of energy, the Substantia Nigra, but the arrangement is after the so called vital nucleus; in comparable way in which childhood lens nucleus forms and envelop around embryonic and fetal nucleus.

Memory seems to be organized in the same way.

Oldest and therefore most fundamental memories for life, are located as close as possible to the Substance Nigra, which is the source of energy. Evolutionary mind makes little sense without those memories, as heartbeat, respiratory frequency, reflex movements, etc., without them life is not possible.

Then have the memories that, although not are so indispensable, they are necessary, as are the mechanisms that control the voluntary contraction and relaxation muscle, and since evolutionary mind appeared after, then is are more away of the source of energy (Substantia Nigra) and around the structures that appeared before, complementing the function.

We can match the embryonic nucleus of the lens with the primitive or vital memory, something like the operating system of the organism. The embryonic nucleus of the eye lens, would be those memories that control processes that are important, but not as vital as it is the language, some aspects of it swallowing; reflex movements, etc.

The lens childhood nucleus would correspond to the memory of what we learn at this stage of life, to walk, to run; to speak, the affective relations; the development of the personality, etc. The core youth corresponds to what we learn during that stage of the life, relations social more complex, conduct sexual; etc. And it is further away from the Substantia Nigra.

Apparently, the sequence is immutable because one does not form if there is no preceding memory or kernel. In the end, it is a whole. We are a whole interconnected in a very complex way, but with a primary need in common: energy.

Finally, we have the nucleus of adult formed late in life. And what could correspond to the recent memory which is which would be more distant from the source of power of the CNS and closer to the cerebral cortex.

For this reason, when the generation and distribution of power are altered for any reason, more distal parts to the Substantia Nigra are the first to show signs of functional impairment. I mean, for example, the recent memory.

As the generation and distribution of energy continues impoverishing, the structures close to the Substantia Nigra also will begin to give signals of deterioration; such as disorientation, unfounded fears, aggressiveness, etc.

Processes and structures related to memory are organized from the melanin, which evolutionary mind makes sense. Because while nearest are to the Substantia Nigra, most important they are for life and further away from the self-conscious, at the same time are more distant to the phylogenetically recent cerebral cortex structures.

Conversely, the structures closest to the cerebral cortex are present on the conscious and less on the unconscious. Although the relationship is not linear since we are interconnected in a highly complex way result of billions of years of evolution, but in the end, depends on a substantive part of the generation and distribution of chemical energy.

The visual pathway is a good example. Pupillary reflexes are almost completely involuntary; binoculars movements are partially voluntary. And the vision, despite having a significant contact with the brain cortex through the optical radiation; has relatively little of voluntary. Thus, for example, in physical and chemicals phenomena that give rise to the visual sensations, at the level of the retina, in cones and rods; the will does not have anything to do; unless we voluntarily closed the eyelids.

The emotional ties are another example, while closer to the source of energy (Substantia Nigra), stronger and deeper at the same time. 


\section{Korsakoff's Syndrome}

First described in 1887 [5], disease develops after chronic alcohol abuse, and is characterized by symmetrical brain lesions along the walls of the third and fourth ventricles as well as in the cerebellum and cerebral cortex. The disease produces a spectrum of cognitive deficits and moderate to severe loss of memory.

This disease is interesting for us, because alcohol molecule forms covalent bonds with receptors involved in generation and distribution of melanin's energy. Thereby, patients with Korsakoff syndrome have cognitive and other clinical deficits not shared by other amnesic groups. These individuals commonly exhibit apathy, blandness, lethargic indifference, vacuity of expression, loss of initiative, placidity, etc. this is: a generalized failure.

Two amnesic syndromes, diencephalic amnesia and bitemporal amnesia are clinically different, but in terms of generation and distribution of energy from neuromelanin, the processes are impaired in severe form when even diencephalic structures are damaged, and less affected when temporal lobes have dysfunctions.

After decades of experimental work, the understand the basic memory defect in amnesia remains as matter so hard to settle, hampering the study of the neurology of memory. Little or nothing is known about the neurobiology of learning and memory, but it is out of discussion that all involved biochemical events need available chemical energy provided by the neuromelanin water's dissociation. In spite, anatomic location of specific brain structures related to learning and memory, the levels of chemical energy are fundamental for the right fitness of the groups of involved neurons.

\section{Melanin and the Optic Nerve}

Melanin is present in all tissues of the body. It is in skin, hair, the uveal tract of the eye, the retinal pigment epithelium, cochlea of the inner ear, the leptomeninges, also in areas as vaginal mucosae, oral, pulmonary and digestive tracts. Thereby the optic nerve is no exception.

The unsuspected intrinsic property of melanin dissociates the molecule of water, such as chlorophyll in plants; It was detected during an observational study, descriptive about the morphological characteristics of the vessels blood that enter and leave the optic nerve, and its possible relationship with the three leading causes of blindness in the world: macular degeneration relating to age, diabetic retinopathy and glaucoma.

The study began in 1990 and ended in 2002. Studies of retinal angiography of almost 6000 patients were reviewed. The main variable in the study were the morphological details of vessels entering and leaving of the optic nerve. A few weeks of having started, the omnipresence of melanin in all patients who we were (Figure 9), we had drawn attention, so we include it as a variable in the study.

The insistence of nature in place melanin in the almost six thousand patients who were included in the study, was another factor to consider. Melanin which is located inside the eye, is traditionally credited role by absorbing the excess of light that penetrates the eye so that is not reflecting on the inside and the image formed has higher quality. But we began to detect blood vessels tend to move away from the melanin (Figure 10).

Melanin seemed to have an anti-angiogenic effect recurring with amazing regularity, constituting an axiom. What remained was to explain it. They were several years of experiments and literature reviews, in which we were discarding possible explanations, such as anti-angiogenic factors, for example.

Around 1998, we began to detect significantly elevated levels of oxygen in pigmented tissues, which explained the anti-angiogenic effect of melanin. But now we had the problem of explaining such elevation.

It took another 4 years, until, in early 2002, and after ruling out the presence of other molecules able to donate oxygen so constantly that we understood that melanin had the unexpected ability to dissociate the water molecule, as the chlorophyll in plants.

\section{CONCLUSION}

The research about the plot of biochemical processes involved in the biology of learning and memory, 


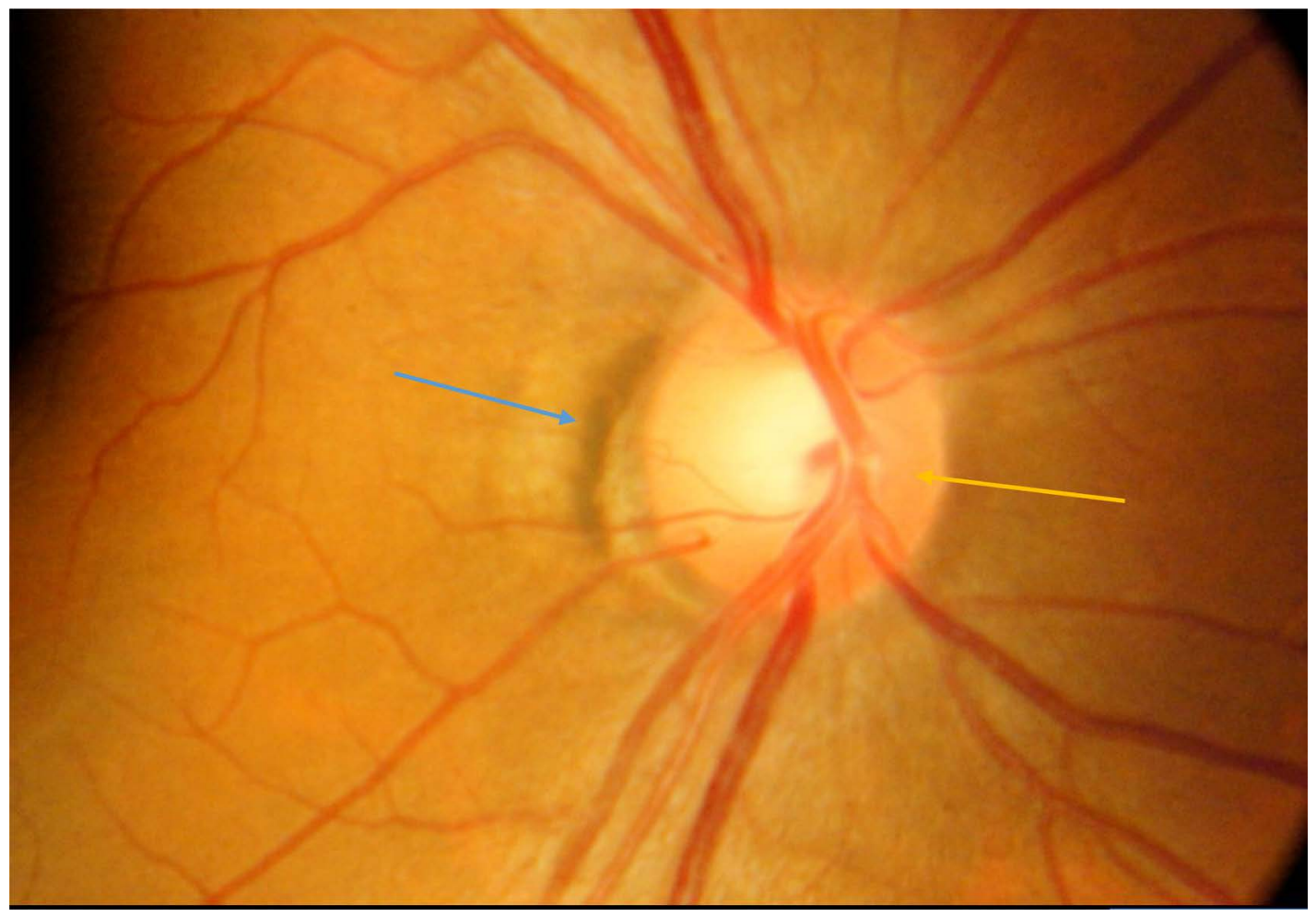

Figure 9. The ever presence of melanin (blue arrow) nearby the optic nerve (yellow arrow) it struck us powerfully.

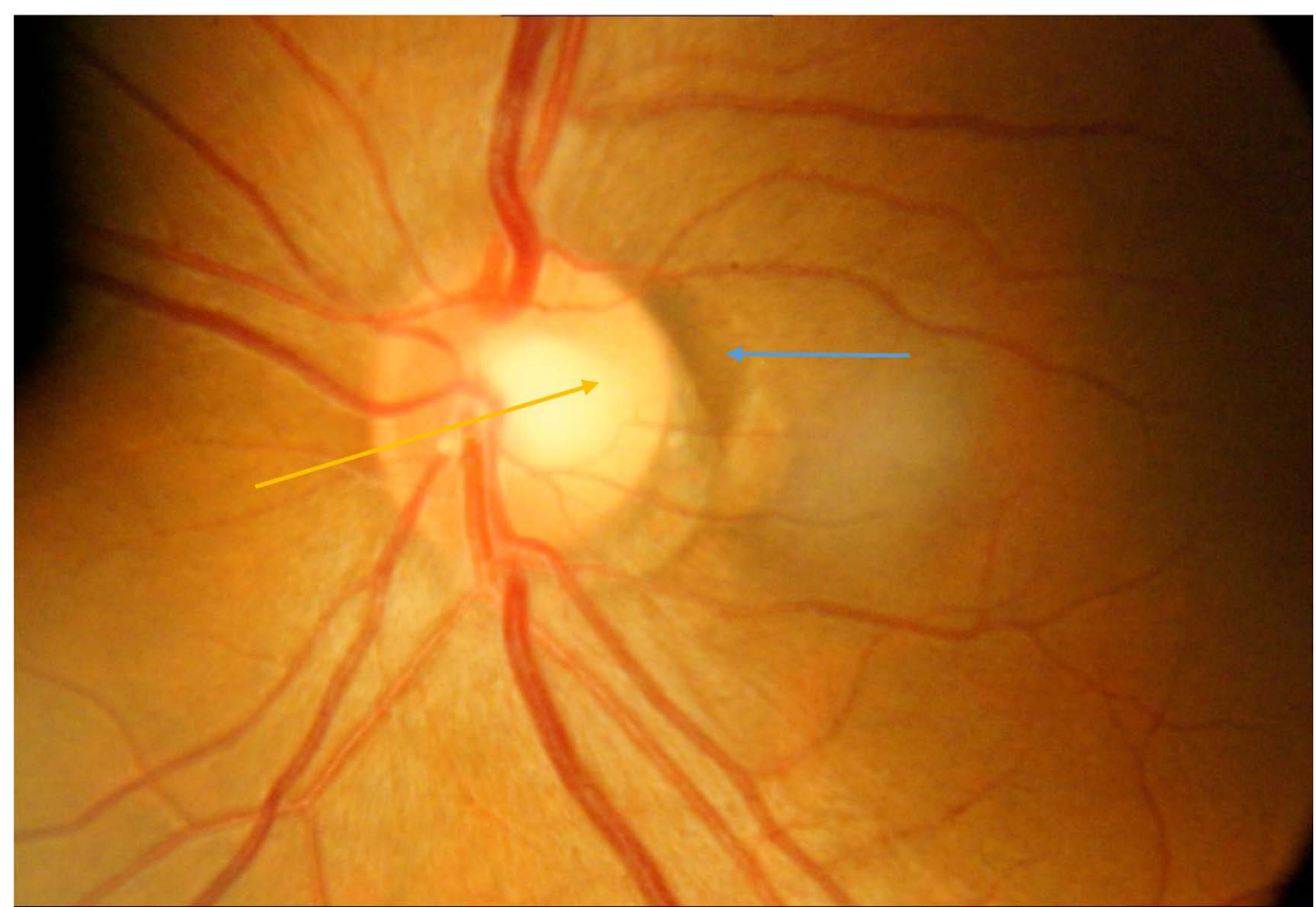

Figure 10. The area where melanin is most concentrated (blue arrow), the blood vessels of the optic nerve tend to be significantly less in number and size (yellow arrow). 
in all its forms, has not considered the generation and distribution of energy needed to power all and each one of them in precision and accuracy happens normally.

Whether the energy of the glucose or the dissociation of water by the neuro melanin, in the work of research on the subject, published in the different scientific journals; different processes are usually mentioned, such as synaptogenesis, neurogenesis; collateral sprouting, receptor adaptations, synaptic depression, facilitation, dendritic growth, intellectual function, dementia, amnesia, etc.

But energy, defined as everything that produces a change, it's never mentioned. The very first step of life, and thereby its origin, is explained by the unsuspected intrinsic property of melanin to transform visible and invisible light into chemical energy through the dissociation of the water molecule, as chlorophyll in plants.

It seems that researchers start from some process that they are interested, and hence build up a theoretical scaffolding surrounded by an aureole of science and zero clinical results. We must keep in mind that, in biology, nothing makes sense except considering evolution. Therefore, to review or experimentation at some isolated part of the whole, it is necessary to start at the very beginning, and that we now know is the generation and distribution of energy by melanin. Otherwise they are measuring in a very precisely way some prevalent bias [6], but the reality is far.

There are data in the literature for more than 100 years pointing that light has an important, even decisive effect in different biological processes, but apparently was not repaired in them or they were misinterpreted.

We will then point out some:

"The exchange of oxygen which takes place between the atmosphere and the blood cannot be adequately explained by diffusion alone." "... gave an oxygen tension considerably above that of the air ..." In carrying out the titration (of $\mathrm{CO}_{2}$ in blood) we avoided bright light, which, from the experiments given in our previous paper, might have appreciably affected the results" [7].

"Levels of acetylcholinesterase activity can be made to vary in response to the presence or absence of sunlight in a system that can be considered as a model for photoperiodic processes found in nature" [8].

"Melanin could act as intrinsic semiconductor and may de-excite certain biological molecules by converting electronic energy into heat" "The melanin pigment is found in all living kingdoms and under different structures and forms" "Melanin acts as Thermoregulation system" "Melanins can scavenge very efficient hydroxyl radicals or molecular oxygen".

"Melanin can transform light energy into electrical energy or heat" "Living organisms are permanently exposed to ionizing electromagnetic radiations when the high energy photons interact with molecules which initiate radiation damages" "For mammals and birds, the Gloger's rule applies: they tend to have black coloration in wet tropics to be brown in desert areas and to be light colored in Artic zone" [9].

Future Study and Remaining Questions

Melanin dissociates water in a surprisingly accurate manner, the reaction products of the dissociation-from liquid to gas-always are molecular hydrogen, molecular oxygen, and to reform the water-gas to liquid-are 4 high-energy electrons by each of two reformed water molecules. The time required for melanin to dissociate the water molecule is in order of the Pico and Nano-seconds.

Melanin absorbs light energy and dissipates it in a unique way: separating the water molecule. What follows is integrating it into the study of basic mechanisms of the biology of learning and memory.

Schemes that are implemented from now on in relation to the functioning of the structures related to memory and learning, must be considered that the brain obtains energy through molecular hydrogen, which is a gas, and that the building blocks of biomass nervous tissue obtained them through the blood.

The molecular hydrogen, is the lightest gas, so the anatomical location of the pigmented structures is below the basal ganglia. But when ascending the molecular hydrogen, this will be captured by these structures.

The biochemical processes involved in memory and learning will remain a mystery, as millions of years of evolution far outweigh our ability of abstraction. His study continued forward, but now we know that the first step involved in these processes is the available energy that comes from the melanin. 
The processes of the body are surprisingly accurate, and the CNS is no exception. Random does not intervene or almost does it, but it is necessary that the system has the same levels of energy that has taken over all the evolution, during all of creation. When the coming energy levels of melanin are altered e.g. by contaminated water, polluted air, pesticides, herbicides, fertilizers, metals, plastics, alcohol, anesthetic agents, fluctuations in temperature, blows, drugs, etc., then the body's biochemical processes are altered unpredictably and the role of chance starts to be relevant. Hence the difficulty of Histochemical level understanding alterations involved in the effects of memory and learning.

\section{ACKNOWLEDGEMENTS}

This work was supported by Human Photosynthesis ${ }^{\circledast}$ Research Center.

\section{REFERENCES}

1. Solis Herrera, A., Esparza, M.C.A., Ashraf, G.Md. Zamyatnin Jr, A.A. and Aliev, G. (2015) Beyond Mitochondria, What Would Be the Energy Source of the Cell? Central Nervous System Agents in Medicinal Chemistry, 15, 32-41. https://doi.org/10.2174/1871524915666150203093656

2. Brenner, S. (2014) Parkinson's Disease May Be Due to Failure of Melanin in the Substantia Nigra to Produce Molecular Hydrogen from Dissociation of Water, to Protect the Brain From Oxidative Stress. Medical Hypotheses, 82, 503. https://doi.org/10.1016/j.mehy.2014.01.013

3. Kandel, E.R. (2001) The Molecular Biology of Memory Storage: A Dialogue Between Genes and Synapses. Science, 294, 1030-1038. https://doi.org/10.1126/science.1067020

4. Solís-Herrera, A., Arias-Esparza, M.D.C. and Solís-Arias, M.P. (2013) Energy Production, the Main Role of Melanin in the Mesencephalon. Journal of Applied Medical Sciences, 2, 11-20.

5. Squire, L.R. (1982) The Neuropsychology of Human Memory. The Neuropsychology of Human Memory, 5, 241-273. https://doi.org/10.1146/annurev.ne.05.030182.001325

6. Ioannidis, J.P.A. (2005) Why Most Published Research Findings Are False. PLOS Medicine, 2, 696-701. https://doi.org/10.1371/journal.pmed.0020124

7. Haldane, J. and Smith, L. (1896) The Absorption of Oxygen by the Lungs. From the Pathological Laboratory, Queen's College, Belfast.

8. Bieth, J., Wasserman, N., Vratsanos, S.M. and Erlanger, B.F. (1970) Photo Regulation of Biological Activity by Photochromic Reagents, IV. A Model for Diurnal Variation of Enzymic Activity. Proceedings of the National Academy of Sciences, 66, 850-854. https://doi.org/10.1073/pnas.66.3.850

9. Césarini, J.P. (1996) Melanins and Their Possible Roles through Biological Evolution. Advances in Space Research, 18, 35-40. https://doi.org/10.1016/0273-1177(96)00025-7 